\title{
Dominant factors determining differences of COVID-19 fatalities between India and other large-population regions
}

\author{
Sudhakar Yarlagadda ${ }^{1,2}$ and Satyaki $\operatorname{Kar}^{3}$ \\ ${ }^{1}$ Academic Council, Visva-Bharati Univ., Santiniketan, West Bengal \\ 2 Saha Institute of Nuclear Physics, Kolkata, India and \\ 3 Department of Physics, Aghorekamini Prakashchandra \\ Mahavidyalaya, Bengai, West Bengal 712611, India
}

(Dated: July 3, 2020)

\begin{abstract}
We analyze the Covid-19 mortality scenario in India and compare it with those in other largepopulation regions such as Asia-excluding-China, Africa, European Union, South America, and USA. We compare existing fatality data and offer an interpretation for low fatality based on immunity due to endemic malaria and TB. We identify the hot climate in the past summer as a possible cause for low death count in southern-hemisphere countries without endemic malaria and TB. We also make India-specific observations for easing the lockdown and estimations for the time required to attain herd immunity. Whatever optimism we present should be viewed as a guarded optimism. There should not be room for complacency.
\end{abstract}

Corresponding author's email: y.sudhakar@saha.ac.in

Keywords: Covid-19, Indian scenario, Inherent immunity, Hot climate, Herd immunity 


\section{INTRODUCTION}

The World Health Organization (WHO), on 30/Jan./2020, declared the novel coronavirus outbreak (2019-nCoV) to be a Public Health Emergency of International Concern (PHEIC) ${ }^{1}$. Next, when the virus spread internationally, on 11/March/2020 the WHO declared COVID-19 a "pandemic" 213. Although initially the scenario regarding the primary driver of transmission was unclear $\frac{45}{45}$, it now appears that airborne transmission is the dominant route for the spread of COVID-196; the incubation period ranges between 2 and 14 days ${ }^{78}$ during which even pre-symptomatic carriers can infect others. The COVID-19 pandemic presents an urgent challenge to understand and formulate a strategy to deal with infection spreading and fatalities. Rising up to the need, there are some number of sources for data on infections and fatalities for various affected countries/regions in the world 11 .

There is a large variation in the number of tests-per-million done by various countries to identify COVID-19 patients. Some of the determining factors are the strategies for testing, availability of testing kits, experienced personnel to handle the testing, etc. Especially when the tests are done based on symptoms shown and when the number of asymptomatic cases is several times that of the symptomatic cases (as shown by the COVID-19 data), the number of detected cases will certainly be much smaller than the actual number. Furthermore, initially when there is no lockdown and there is an exponential growth in the number of infected people, the number of tests conducted may not be able to keep in pace with the growing number of infections. Thus it is important to realize that the number of infected cases reported by various countries will not be a good measure of the spread of infection in those countries even for comparative purposes. On the other hand, the number of fatalities is likely to be not in serious error as the detection of death due to severe acute respiratory syndrome is not difficult to diagnose. The main purpose of the present paper is to do a comparative study of the fatalities due to COVID-19 in India and other large-population (i.e., greater than 300 million population) regions such as USA, South America, Africa, European Union, and Asia-excluding-China. Based on the comparative analysis, we aim to offer a strategy of how India should deal with the COVID-19 situation. Part of the motivation for this work stems from the predictions of large-scale deaths in India by groups such as Eili Klein et al! 12 .

There can be several factors that could determine the deaths due to COVID-19. We 
present some of them below:

1) Intrinsic immunity of populations (such as Indian, African, etc.) may arise due to extensive exposure to harmful/killer pathogens corresponding to Malaria, Tuberculosis (TB), diarrheal diseases, etc. Moreover, the regular usage of BCG vaccination in India, Africa, etc. for some decades could have provided strong immunity against disease causing organisms such as the COVID-19 virus (see Ref. 13 for a justification).

2) Higher temperatures and sunny weather impact on the fatality. Low vitamin D levels have been associated with a number of factors including less exposure to the sun. It has been reported recently that there is a significant relationship between vitamin $\mathrm{D}$ levels and number of COVID-19 cases and mortality 14 .

3) Age of population affecting the vulnerability to death due to COVID. It is has been found that death rates are higher among older people (see data from New York at https://www.worldometers.info/coronavirus/coronavirus-age-sex-demographics/).

4) Urbanization can also have an effect on mortality. Increased connectivity and increased population density, by-products of urbanization, could have adverse consequences such as increase in transmission of COVID. On the other hand, usually urban areas have better health-care facilities than rural areas; this implies urbanization can have a favorable effect. 5) Food habits can offer better resistance to COVID related deaths. Large amount of literature exists (in Indian systems of medicine such as Ayurveda) on the clear benefits of Indian spices in increasing immunity. For instance, turmeric, a common ingredient of various Indian dishes, is known to have antioxidant, anti-inflammatory, anti-bacterial, and wound-healing properties 15 .

6) Cumulative affect of pollution can determine the susceptibility to COVID death. It has been found that particulate matter (such as carbon) has significant effect on bacteria which are central to ecosystems in humans and in the natural environment $\frac{16}{16}$.

7) Wild life destruction (such as hunting for sport, systematic elimination of large animals, etc.) could play a role in affecting human immunity to diseases such as COVID. Upsetting the delicate balance of nature is likely to have an impact on the natural environment and consequently on human immunity.

Additionally, government interventions such as lockdowns have a crucial role in containing the community transmission of COVID and in determining the fatalities ${ }^{17} 18$.

Given the number of variables that can affect the death count due to COVID and the 
limited time over which the data is available, we find it better to identify important correlations and useful global scenarios using the available data rather than perform detailed mathematical modeling of a complex problem. In this connection, it should be noted that important microscopic details such as $R_{0}$ (the reproduction number) $\frac{19}{19}$ are poorly determined; furthermore, the evolution of the virulence of the virus also seems to be not well understood 20 .

In this paper, we focus mainly on the effect of the first three factors (i.e., the effect of endemic diseases, age of population, and sunny climate) on the number of COVID deaths; we relegate an analysis of the remaining four factors to a later study.

\section{ANALYSIS}

The following are some points considered in our analysis:

1) Counting the number of infected cases for comparison between countries does not really make much sense as the number of cases increases with the number of tests performed. Testing, based on symptoms, is not indicative of the number of infected as ICMR seems to be claiming that as high as $80 \%$ of the infected could be asymptomatic.

2) Contrastingly, the number of deaths is a much more reliable measure. One cannot be wrong by more than a factor of two even if there is a large amount of misdiagnosis. At any rate, finally what matters is the number of deaths.

3) It is generally believed that, because of various factors (some of which are mentioned below), Indians have some level of immunity protection. Based on information such as the world maps of TB endemic countries and malaria endemic countries (see Figs. 1 and 2), we see that the countries most affected by TB and malaria are, in general, turning out to be the least afflicted by COVID-19 (for COVID-19 information, see Refs. 10 and 11). Thus, one can draw the conclusion that Indians, Africans, etc. may have certain immunity.

It is also quite possible that hot sunny weather may help Indians as vitamin D production is greater in sunny conditions. If hot summer offers more protection, then lockdown in March and April helps not only in increasing health-care capacity but also in protecting till favorable summer-time arrives.

4) Modeling, based on climate and immunity, is not easy. However, one can conservatively draw conclusions based on existing trends. Present death rates do indicate that, in contrast 
to other regions with large populations, India has low death rates. Our method of comparison is as follows.

We compare India and other regions with large populations (i.e., populations greater that 300 million) such as Asia-excluding-China (AEC), Africa, European Union (EU), South America (SA), and USA. Starting point of comparison is when India crossed 100 deaths and when other regions crossed the corresponding scaled number of deaths given by $100 \times$ (population of region)/(population of India).

From data in https://ourworldindata.org/grapher/total-covid-deaths-region ${ }^{11}$ (also see Fig. 3):

India crossed 100 deaths on Apr./6; USA recorded 26 deaths on March/10; European Union reported 37 deaths on March/2; and South America registered 37 deaths on March/22.

Countries with indigenous cases in $\mathbf{2 0 0 0}$ and their status by 2017 Countries with zero indigenous cases over at least the past 3 consecutive years are considered to be malaria free. All countries in the WHO European Region reported zero indigenous cases in 2016 and again in 2017. In 2017, both China and El Salvador reported zero indigenous cases. Source: WHO database.

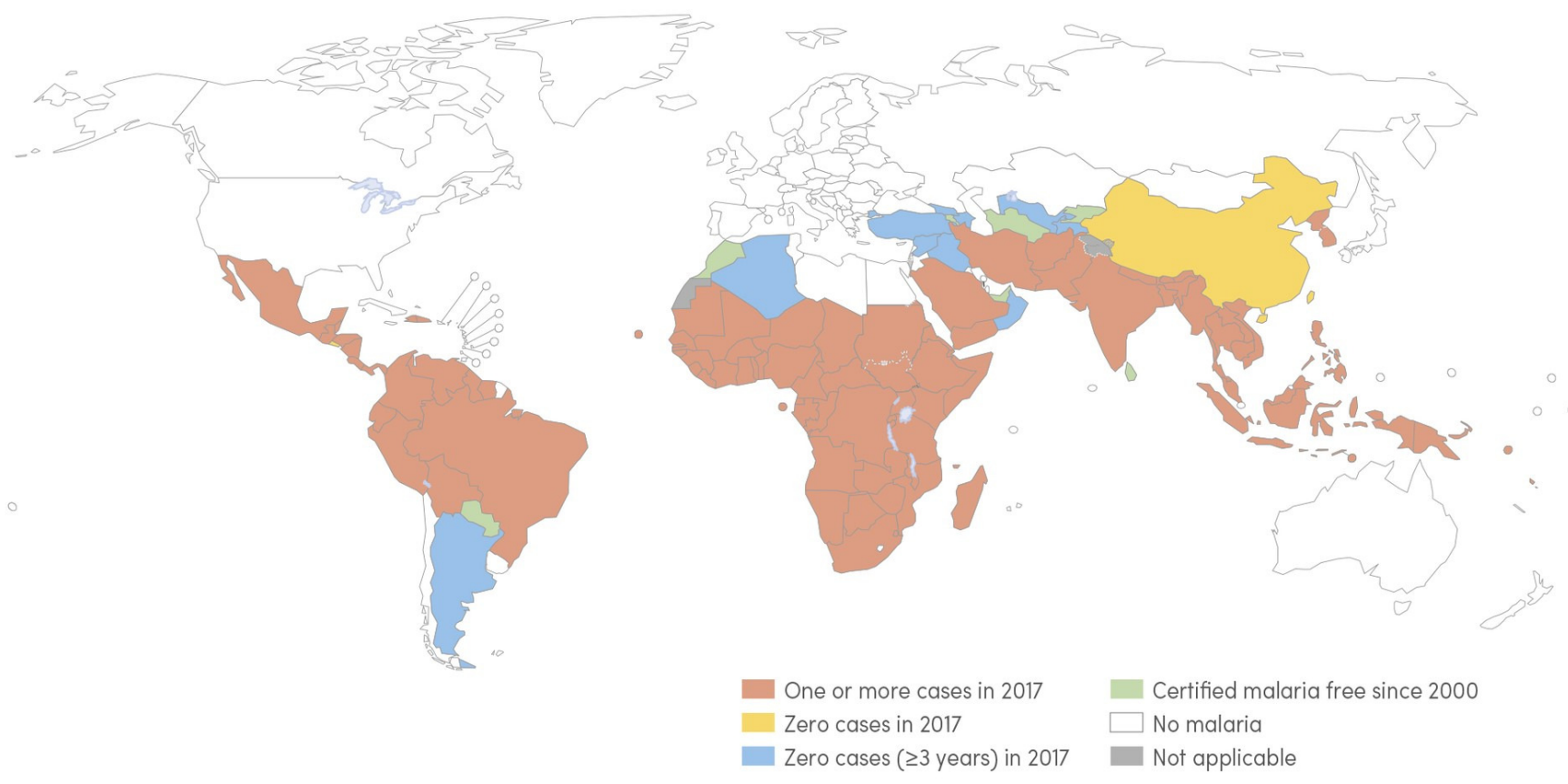

WHO: World Health Organization.

FIG. 1. (Color online) World map of malaria endemic countries 21 . 


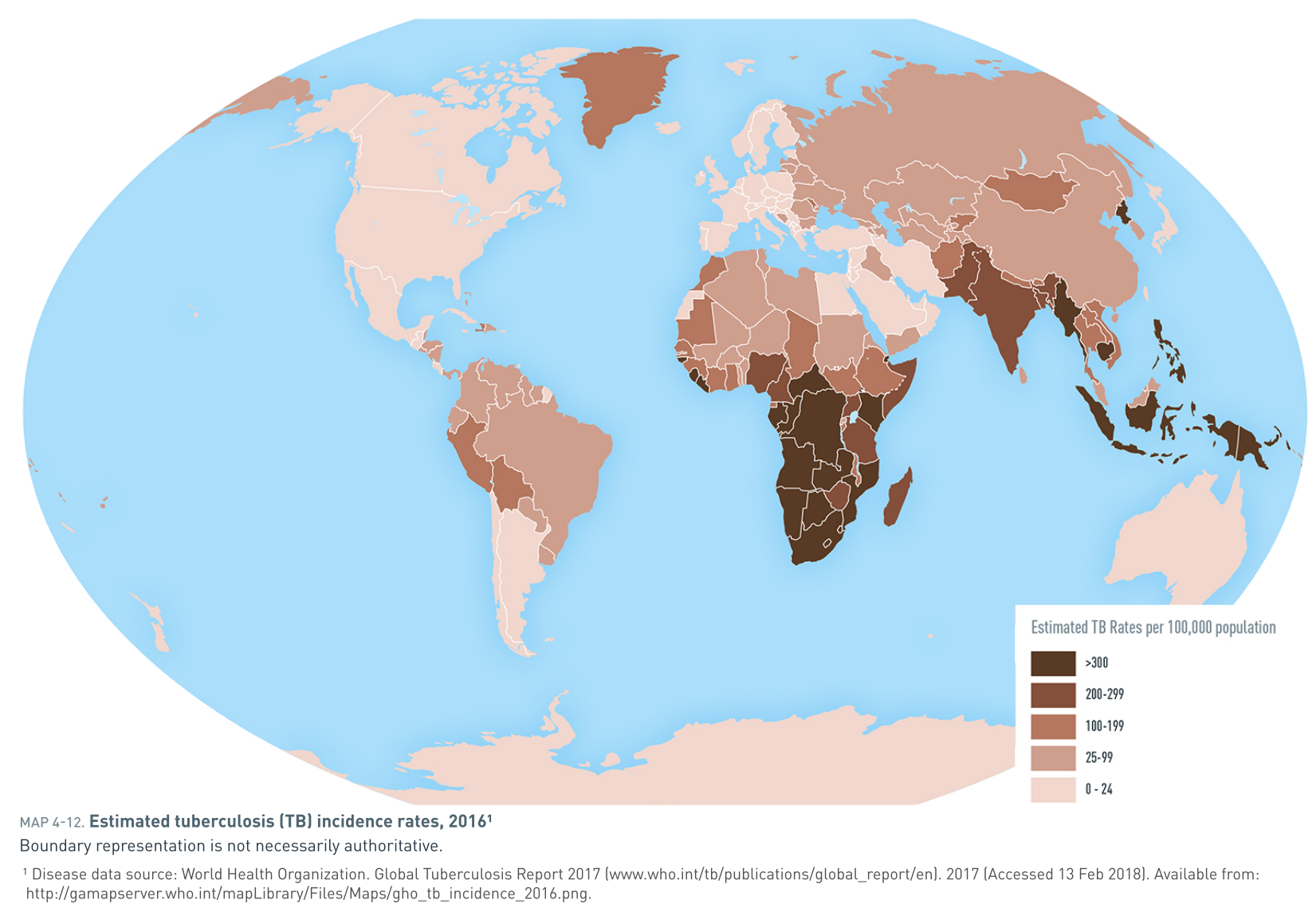

FIG. 2. (Color online) World map of TB endemic countries 22 .

On adding 79 days to the starting point of each region, we note the following:

Till June/24, total number of deaths in India was 14476; till May/28 number of fatalities in USA was 100442; till May/20 death count in European Union was 122659; and till June/9 death tally in South America was 51157.

It is clear, from Fig. 3(a), that total number of deaths in India are about $1 / 7$ those of USA, less than 1/8 those of European Union (EU), and less than 1/3 those of S. America. Furthermore, more contrastingly, Fig. 3(b) reveals: per-capita fatality in USA is approximately 29 times that of India; per-capita death count in EU is 26 times that of India; and per-capita death tally in South America is 11 times that of India.

In spite of the fact that people in USA have about 12 times the area-per-person as Indians, people of EU have more than 4 times the area-per-person as Indians, and South American's have more than 16 times the area-per-person as Indians (see Table. I), India seems to be doing much better; we believe that this is probably indicative of immunity based on usual 


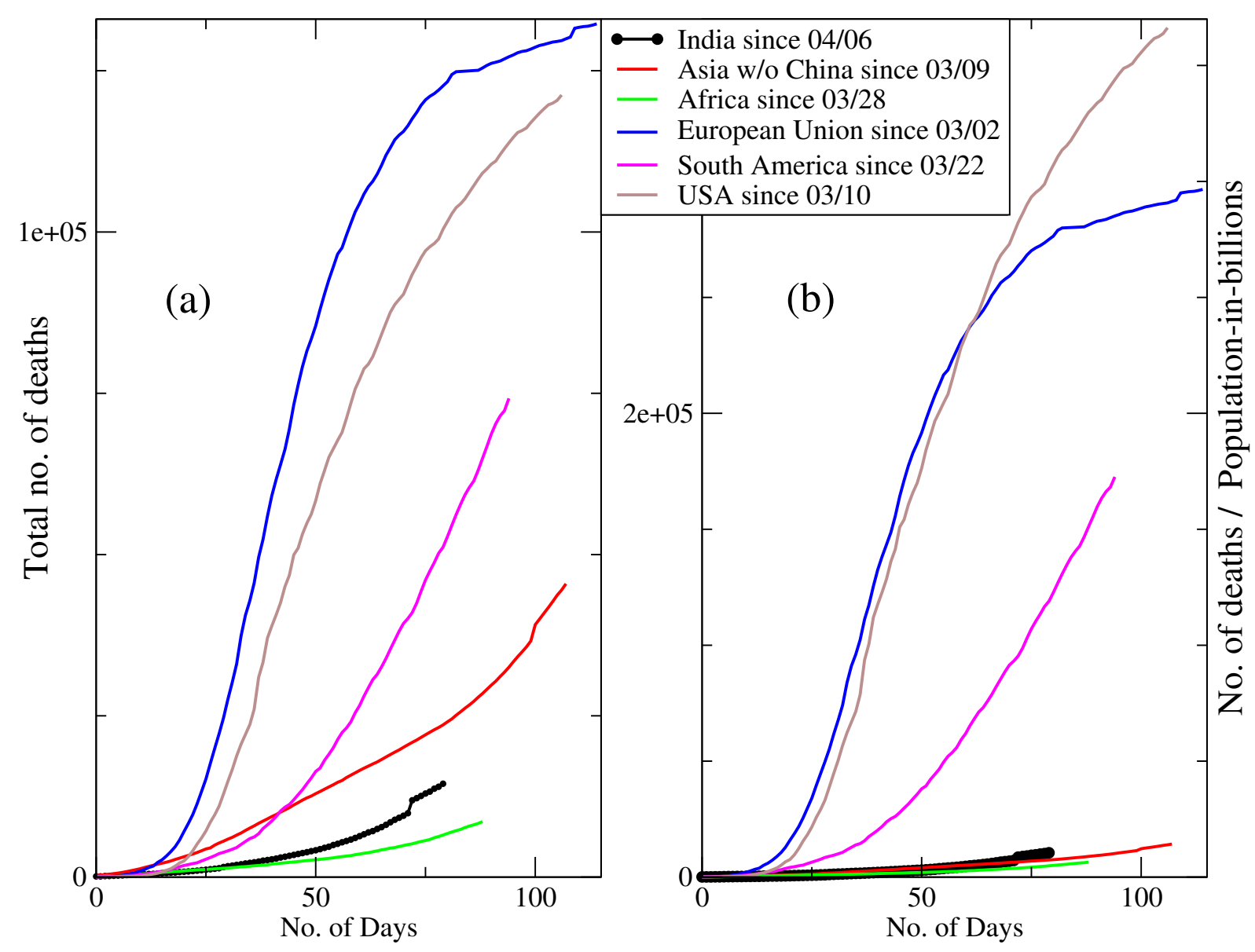

FIG. 3. (Color online) Total fatalities as reported in "our world in data"11. Starting point of comparison is when India reported 100 deaths and when other regions reported the number of deaths given by $100 \times($ population of region)/(population of India). Plots are for (a) Total number of deaths and (b) Number of deaths per billion of population.

prevalence of malaria and TB (see Figs. 1 and 2).

The populations of the African continent and India are roughly the same (see Table I). Their trajectories for total fatalities (see Fig . 3) are not too different; in fact, Africa recorded roughly only about half the number reported by India. Since, like Indians, Africans also have immunity, the death rates of these two regions can be, to some extent, comparable. However, since Africa has about 10 times the area of India, the area advantage probably helped Africa keep the number of deaths lower. Lastly, Asia-excluding-China, after 79 days from the starting point of March/9 (when it reported 261 deaths), had a death tally of 23594 which is not much smaller than 33525, i.e., the Indian number modified in proportion to the population $(14476 \times 3196 / 1380 \approx 33525)$. Furthermore, in Asia-excluding-China the area 


\begin{tabular}{|c|c|c|}
\hline Region & Population (Millions) & Area (Million Sq. Kms.) \\
\hline India & 1380 & 3.29 \\
\hline Asia w/o China & 3196 & 34.98 \\
\hline Africa & 1335 & 30.37 \\
\hline European Union & 446 & 4.48 \\
\hline South America & 430 & 17.84 \\
\hline USA & 331 & 9.83 \\
\hline Argentina & 45.1 & 2.78 \\
\hline Australia & 25.4 & 7.69 \\
\hline New Zealand & 4.8 & 0.27 \\
\hline
\end{tabular}

TABLE I. Comparison of populations and land areas of various regions (based on Wikipedia)

per-person is more than 4 times that in India; perhaps this could explain the difference in the per-capita death count.

It is also interesting to compare one of the best scenario countries in Europe (i.e., Germany) with one of the worst cases in Asia (i.e., Iran). Both Iran and Germany have approximately 82 million people. Iran started being a hotspot before Germany, yet both have reported approximately comparable number of deaths. More exactly, (from data in https://www.worldometers.info/coronavirus/ ${ }^{10}$ ) as on June/24, Iran has had 9863 deaths while Germany reported 8914 deaths. In fact, a reason why Iran has higher number-ofdeaths-per-million among Asian countries could be that, although malaria infections exist in Iran, it has low prevalence of TB.

As regards climate, hot sunny weather seems to help keep the death-count low in countries such as Australia, New Zealand, and Argentina which have come out of summer, fairly recently, in the southern hemisphere; these countries do not have prevalence of malaria or TB. From Fig. 4, it is obvious that the fatality count is low, possibly due to hot sunny climate, even in countries with moderate population such as Argentina and Australia (see Table. I). It should be noted that, in Fig. 4, the starting point for each country is when the first death was reported. This is because the number of deaths given by $100 \times($ population of country/population of India) is of the order unity. 


\section{CONCLUSIONS AND WAY FORWARD}

We feel that the age of the population, while being partially responsible for a lower fatality, is not a decisive factor in explaining the low mortality and offer the following justification. We first note that India has a median age of about 28 years; Africa has a median of about 20 years; Asia-excluding-China has a median less than 31 yrs; USA has a median of 38 yrs; S. America's median is 32 yrs; and EU's median is 43 years. Next, for the sake of argument, let us conservatively assume that all the deaths are from above the median age for India/Africa; since $50 \%$ population of India/Africa is more than 650 million which is

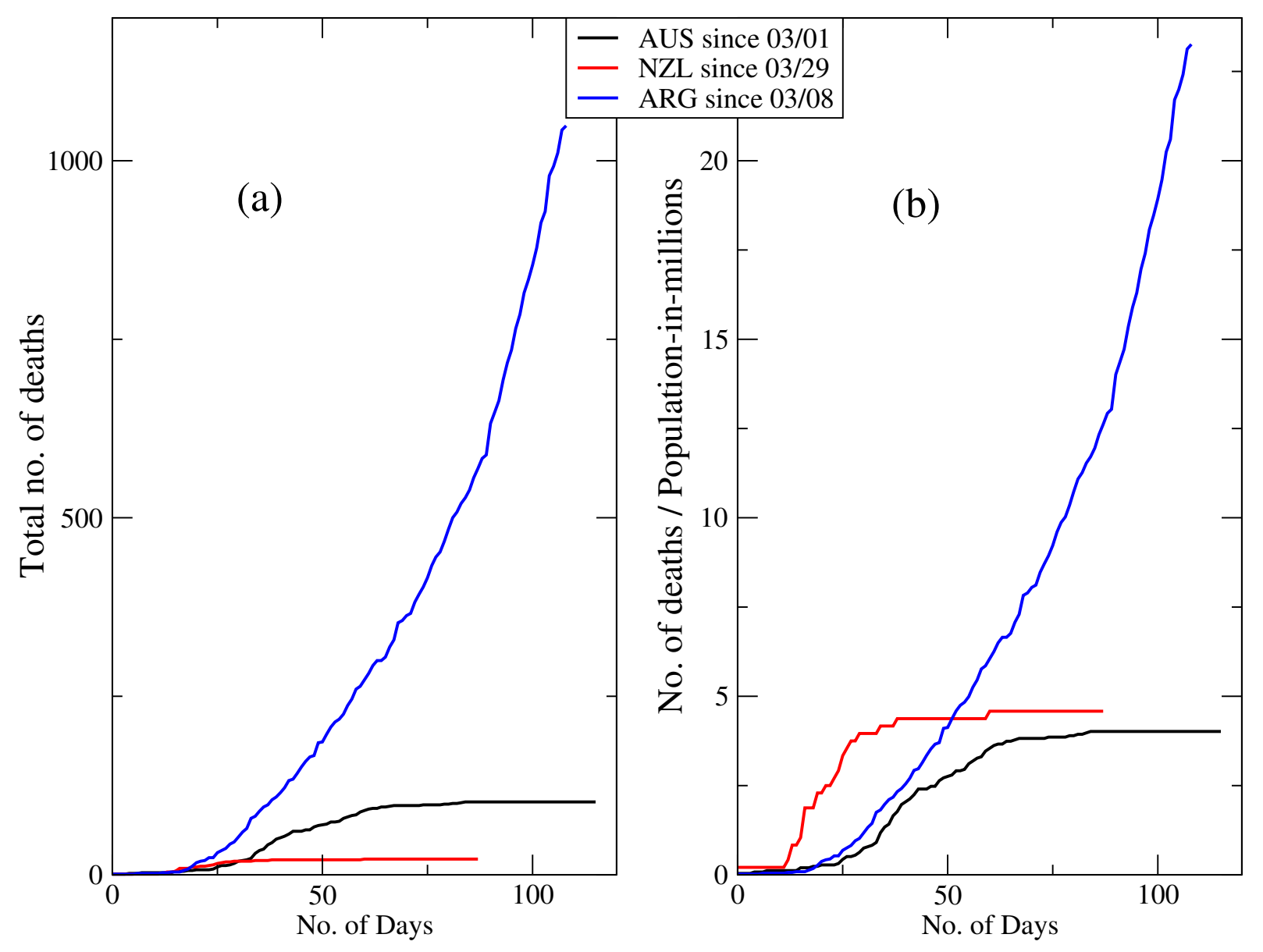

FIG. 4. (Color online) Total fatalities as reported in "our world in data"11 for a few countries (Argentina, Australia, and New Zealand) in the southern hemisphere that do not have prevalence of malaria or TB. The starting point for all the countries is when the first death was reported in that country. Plots are for (a) Total number of deaths and (b) Number of deaths per million of population. 
more than the population of European Union (i.e., 446 million) or South America (i.e., 430 million) or USA (i.e., 331 million), India/Africa are doing much better based on our figure 3.

In summary, we would like to say there seems to be a strong correlation between low fatalities and endemicity of malaria and TB in regions with large populations. We also find evidence that sunny weather could help lower the mortality; however, more data from the summer season in the northern hemisphere is needed to properly validate the relationship with the climate. Next, we will make a few relevant points.

1. One needs caution extrapolating based on existing trends. As always, extrapolation is less predictable than interpolation.

2. India needs to have its own strategy regarding easing the lockdown restrictions; also needs to develop its own plan of action that keeps in mind its people's well being, economy, and lower death count.

3. India needs to develop its own understanding and strategy for generating herd immunity.

4. People most exposed to TB, malaria, etc. may be better suited to generate herd immunity.

The lockdown can be ended (with continued monitoring of the response) based on agegroup vulnerability and only for people without medical conditions such as diabetes, asthma, heart problems, hypertension, etc. For instance, the less vulnerable age group of below 60 years (i.e., more than $88 \%$ population based on 2011 census) may be allowed to come out of lockdown but with strictly observing the practices of wearing masks and social distancing.

Assuming $R_{0}$ (the reproduction number) ${ }^{19}$ is 1.5 , the herd immunity threshold (HIT) is given by $\left(1-1 / R_{0}\right) \times 100$, i.e., $33 \%$ population which is about $1.38 \times 0.33 \approx 0.455$ billion. Assuming that each person takes (on an average) 10 days to infect 1.5 people (on an average), and given that $1.5^{12} \approx 130$ and that $130 \times 4000000$ is 520 million, one can expect to attain HIT in about 4 months. Here we assumed that the existing infected cases are about 40 lakhs (with 400000 being detected while remaining 3600000 being undetected and asymptomatic). Alternately, (based on current trends given in Ref. 23 during lockdown) we conservatively assume that the daily growth rate in number of cases is $4 \%$ and that existing infected 
cases are about 40 lakhs; then, after 4 months from now, the number of infected cases is approximately 0.46 billion which is about the HIT number.

\section{ACKNOWLEDGMENTS}

One of the authors (S. Y.) would like to thank G. Baskaran, B. Chakrabarti, N. V. Chalapathy, M. V. Ramdas, M. Tambe, Ajay Dubey, Rakesh Khanna, and K. B. M. M. Krishna for valuable discussions.

1 World Health Organization, Coronavirus disease 2019 (COVID-19) situation report-10, (2020).

2 A. S. Fauci, H. C. Lane, R. R. Redfield, New England Journal of Medicine 382, 1268-1269 (2020). doi:10.1056/NEJMe2002387.

3 World Health Organization, Coronavirus disease 2019 (covid-19) situation report-51, (2020).

4 S. Flaxman et al., DOI: https://doi.org/10.25561/77731 .

5 C. Rothe et al., New England Journal of Medicine 382, 970-971 (2020). doi:10.1056/NEJMc2001468.

6 R. Zhang et al., www.pnas.org/cgi/doi/10.1073/pnas.2009637117 .

7 S. A. Lauer et al., Annals of Internal Medicine (2020). URL: https://doi.org/10.7326/M20-0504 .

8 N. M. Linton et al., J Clin Med. 2020;9. [PMID: 32079150] doi:10.3390/jcm9020538.

9 E. Dong, H. Du, and L. Gardner, The Lancet infectious diseases, DOI:https://doi.org/10.1016/S1473-3099(20)30120-1 (2020).

10 https://www.worldometers.info/coronavirus/ .

11 https://ourworldindata.org/grapher/total-covid-deaths-region .

12 Eili Klein et al., CDDEP report (March 24, 2020). https://cddep.org/wpcontent/uploads/2020/03/covid19.indiasim.March23-2-eK.pdf .

13 A. Miller et al., medRxiv https://doi.org/10.1101/2020.03.24.20042937 (2020) .

14 P. C. Ilie, S. Stefanescu, and L. Smith, Aging Clin Exp Res 32, 1195-1198 (2020). https://doi.org/10.1007/s40520-020-01570-8 .

15 E. Abdollahi et al., J Cell Physiol. 233, 830-848 (2018).

16 S. J.K. Hussey et al., Environ Microbiol 19, 1868 (2017). 
17 Debraj Ray and S. Subramanian, "India's Lockdown: An Interim Report," May 20 (2020).

18 Pragyan Deb, Davide Furceri, Jonathan D. Ostry and Nur Tawk, "How the Great Lockdown Saved lives", IMF blog, June 2 (2020).

19 Gregg N. Milligan and Alan D. T. Barrett, (2015). Vaccinology: an essential guide. Chichester, West Sussex: Wiley Blackwell. p. 310.

20 Milind Watve, "Covid 19: We need neither vaccine nor herd immunity", May 27, 2020. https://milindwatve.home.blog/.

21 World Health Organization, World malaria report 2018 (see Fig. 1.1).

22 https://wwwnc.cdc.gov/travel/yellowbook/2020/travel-related-infectiousdiseases/tuberculosis .

23 https://en.wikipedia.org/wiki/Timeline_of_the_2020_coronavirus_pandemic_in_India . 